\title{
Putting the 'P' into Phospholipids
}

\author{
Illya A. Fedotenko, Margaret N. Holme, Radu Tanasescu, Pierre-Léonard Zaffalon, and \\ Andreas Zumbueh"*
}

\begin{abstract}
Despite almost hundred years of dedicated research, the synthesis of phospholipids remains a challenge. Here, we briefly review important trends and point out possible future directions that might lead to interesting new probes for the membrane environment. An improved liposome purification and concentration protocol rounds up the article.
\end{abstract}

Keywords: Liposome ultrafiltration · Phospholipid synthesis · Phosphorus chemistry

Phospholipid-based membrane probes are attractive tools in modern biology and an integral part of the NCCR in Chemical Biology. Meeting the needs of biologists while coping with the constraints of lipid biophysics and organic chemistry will lead to interesting fresh designs for reporting molecules. Certainly, adaptable and modular synthetic strategies are welcome and we would like to take the opportunity to review the recent advances in the field of phospholipid research.

\section{Head First or Tails First?}

A typical phospholipid can be built from four different parts: two hydrophobic tails (e.g. acyl hydrocarbons), a hydrophilic head group (e.g. phosphodiesters) and a backbone holding these units together (e.g. glycerol). ${ }^{[1]}$ The synthesis of a phospholipid may start either by first connecting the head group and the backbone or the tails and the backbone (see Scheme 1). Both strategies have their advantages: the tails-first approach allows the use of readily available natural building blocks such as 1,2-dipalmitoyl-sn-glycerol ( $s n$ denotes the stereospecific numbering of the three glycerol carbon units introduced by

${ }^{*}$ Correspondence: Dr. A. Zumbueh University of Geneva

Department of Organic Chemistry

Quai Ernest-Ansermet 30

$\mathrm{CH}-1211$ Geneva 4

E-mail: andreas.zumbueh|@unige.ch

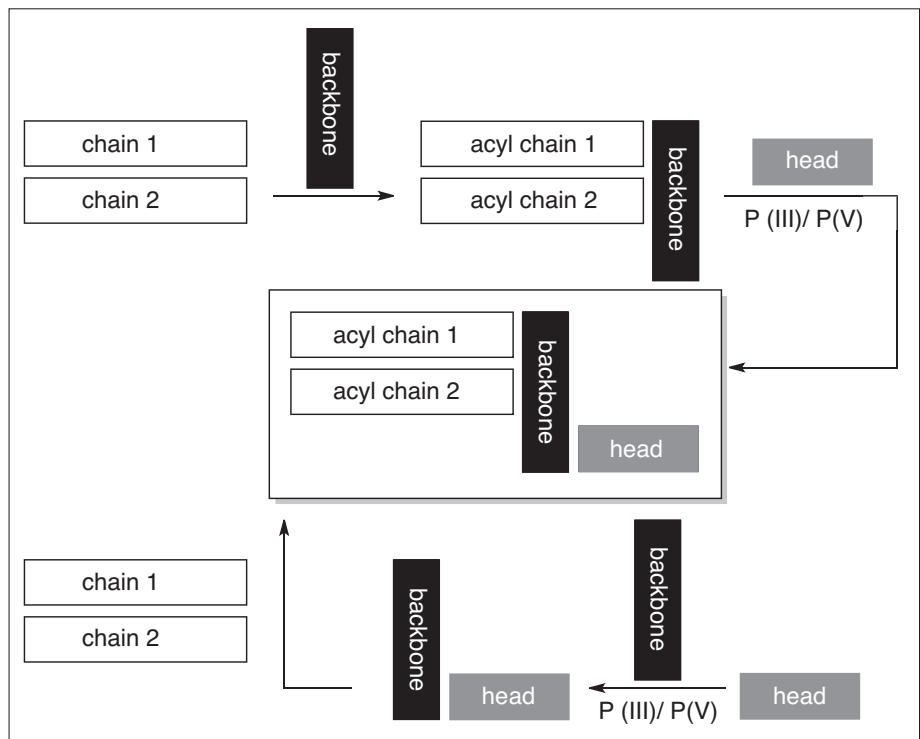

Scheme 1. Schematic workflow depicting the modularity of phospholipid synthesis from four key building blocks.

Hirschmann). [2] However, care should be taken to prevent acyl migration to the 3-position. ${ }^{[3,4]}$ When switching to less natural substitution patterns, such as 1,3-diamido2-propanol, the virtual insolubility of the molecule in any solvent (and thus its low reactivity) puts a premature halt to using the tails-first approach. ${ }^{[5-7]}$

The readily available glycerophosphocholine also shows low solubility in any solvent due to its zwitterionic character and the quarternary ammonium ion and bulky counter ions such as tetraphenyl borate need to be used in order to increase the solubility of the head group. ${ }^{[8]}$ If the charges can be masked by protecting groups until a very late stage in the synthesis, the benefits are not only found in the better solubility of the molecules and their higher reactivity but also in the easier purification on a standard silica gel column. While the tailsfirst approach allows the rapid synthesis of various different head groups from one tail scaffold, the head-first approach allows the rapid introduction of acyl chains of varying length. The phospholipid tail length is linearly proportional to the melting temperature of the molecule and therefore an interesting parameter for tuning liposomal drug delivery systems. ${ }^{[9]}$

\section{Phosphorylation Chemistry Using $P(v)$ Reagents}

Both the head- and tails-first approaches require a phosphorylation step in order to complete the phospholipid. Again two possibilities exist, each with its specific advantages: Phosphorus (v) reagents are generally cheap and easy to handle but they may suffer from modest reactivity. ${ }^{[4]}$ Phosphorus (III) reagents are very reactive, but they are also prone to oxidation and are generally expensive. ${ }^{[10]}$

The very first synthesis of a phospholipid was performed in the 1920s. ${ }^{[11]}$ Grün and Limpächer first phosphorylated choline bicarbonate with diluted phosphoric acid and then coupled this head group to 1,2-distearoylglycerol using such sophisticated techniques as kneading the reagents until they formed a solid that was then boiled in benzene. [11]

In order to extend the lifetime of researchers, more hands-off technologies 


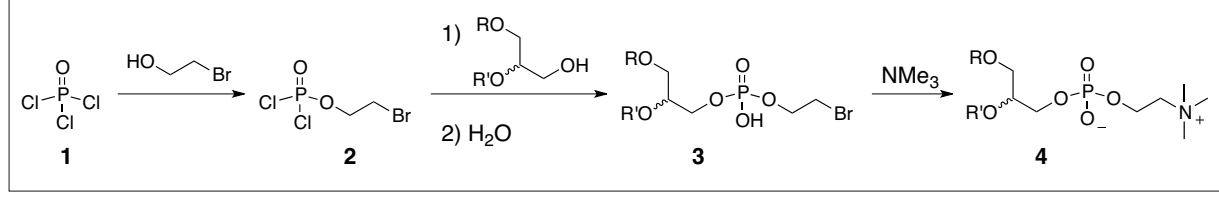

Scheme 2. Rapid synthesis of phosphocholines using the popular bromoethylphosphoric acid dichloride reagent 2 .

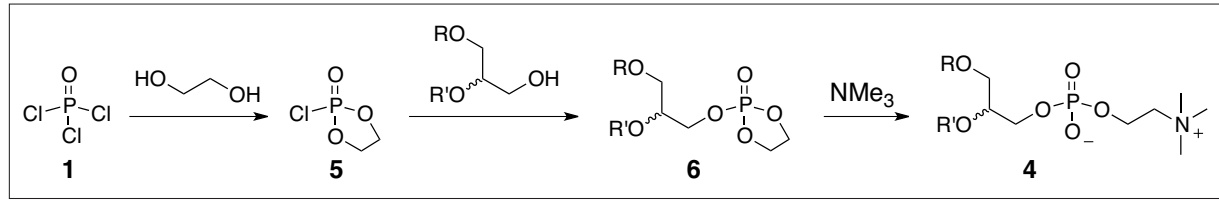

Scheme 3. Phosphocholine synthesis using the 2-chloro-2-oxo-1,3,2-dioxaphospholane reagent 5.

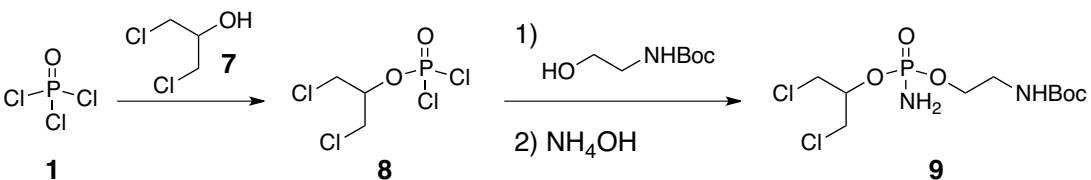

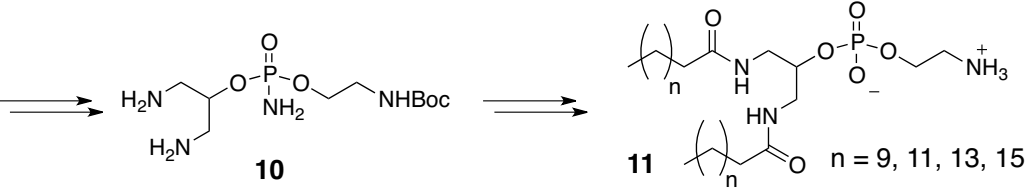

Scheme 4. Synthesis of a library of 1,3-diamidophosphocholines using a phosphorodichloridate reagent 8 .

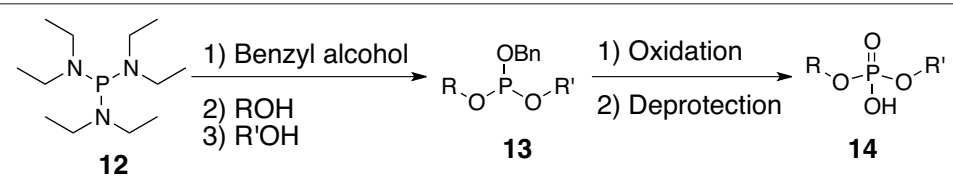

Scheme 5. Stepwise synthesis of a phospholipid starting from hexaethylphosphinetriamine (12).

$$
\text { 15 }
$$

Scheme 6. 2-Chloro-1,3,2-dioxaphospholane (15) is a powerful reagent in phosphorylation chemistry.

were developed and phosphorus oxychloride (1) became a reagent of choice. Rose was the first to demonstrate that primary alcohols could be activated by phosphorus oxychloride (1) and a second alcohol could trap the intermediate in order to produce cephalines (ethanolamines). ${ }^{[12]}$ The lecithines (cholines) were not available using this route since the methylation of the cephaline head group also led to the degradation of ester bonds in the tail-backbone region. Therefore, a new reagent, bromoethylphosphoric acid dichloride (2), was introduced in 1958 by Hirt and Berchtold working at Dr. Wander AG in Bern, and popularized by Eibl in the 1970s (Scheme lating a secondary alcohol and not a more reactive primary alcohol.[4] One of our own solutions to this problem was the use of a phosphorodichloridate (8) whose corresponding phosphate was initially introduced as a flame retardant additive in polymer synthesis (Scheme 4). ${ }^{[7]}$ 1,3-Dichloropropanol (7) was readily substituted with phosphorus oxychloride (1) to give the phosphorodichloridate $(\mathbf{8})$ that could be readily purified by distillation. The introduction of the head group and the tail region was performed on the respective phosphoramidate intermediates 9 in order not to have a charged or zwitterionic molecule that would be more difficult to purify. Using the head group-first strategy we were able to rapidly access a library of amidophospholipids containing alkyl chains of different lengths (11). [7]

However, there are cases where the reactivity of $\mathrm{P}(\mathrm{v})$ is just not high enough and a switch to $\mathrm{P}(\mathrm{III})$ reagents is needed.

\section{Phosphitylation Chemistry Using P(III) Reagents}

One of the first $\mathrm{P}(\mathrm{III})$ reagent used for the synthesis of a glycerophospholipid was reported in 1978 by Shin et al. ${ }^{[17]}$ Hexaethylphosphinetriamine (12) was consecutively reacted with three different primary alcohols (Scheme 5). The asymmetrically substituted phosphine $\mathbf{1 3}$ was then oxidized to the phosphate and deprotected in order to yield the final phosphate diester 14. ${ }^{[17]}$

Chabrier subsequently published a variant of the dioxaphospholane chemistry introducing 2-chloro-1,3,2-dioxaphospholane (15) that was synthesized from trichlorophosphine and ethylene glycol (Scheme 6). After oxidation with $\mathrm{O}_{2}$, the dioxaphospholane (17) was opened using trimethylamine gas in dry polar solvent to yield 19.[18] If the oxidation step was performed using bromine, as proposed by Bittman et al., ${ }^{[19]}$ the choline could be obtained from bromide $\mathbf{1 8}$ without the use of an additional protecting group.

In 1981 Baucage and Caruthers published on the use of deoxynucleoside phosphoramidites, a class of activated phosphites relatively stable towards hydrolysis and air oxidation (Scheme 7). ${ }^{[20]} \mathrm{A}$ key intermediate was chloro- $N, N$-dimethylaminomethoxyphosphine (20) which acted as a monofunctional phosphitylating agent. ${ }^{[20]}$ The high-yielding reaction was catalyzed by acid and phosphoramidite reagents were consecutively also used for the synthesis of various phospholipids. [21-23]

Trying to use a phosphoramidite protocol for the synthesis of phospholipids, we found that not only were the costs of the reagents prohibitively high (and the 


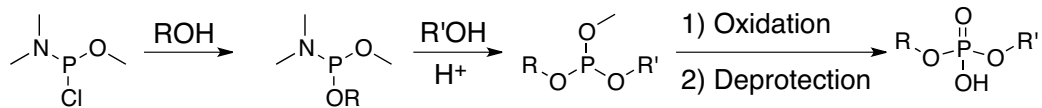

$$
\begin{aligned}
& 20 \\
& 21 \\
& 22 \\
& 14
\end{aligned}
$$

Scheme 7. Phosphoramidite reagents such as chloro-N,N-dimethylaminomethoxyphosphine (20) are also used for phospholipid synthesis.

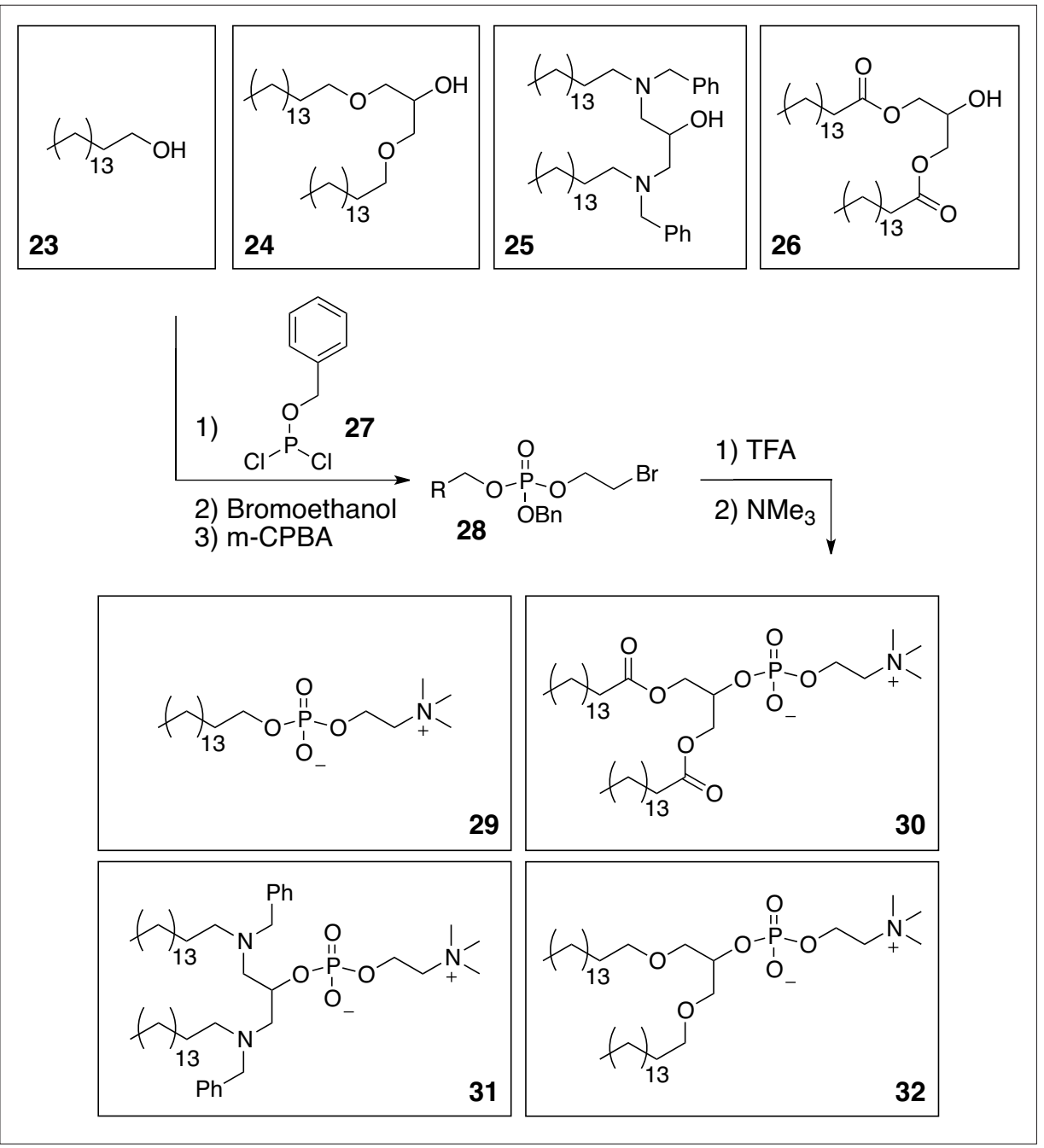

Scheme 8. BODP (27) is a versatile reagent for phospholipid synthesis.

synthesis tedious) but also the resulting intermediates (e.g. 21) could not be purified because they would oxidize immediately to the corresponding stable phospho-amidate. It was interesting to note that phosphorus trichloride itself can be stored for years without having to worry about oxidation. A phosphitylating reagent retaining two chloride functions might therefore show optimal equilibrium between high reactivity and stability towards oxidation. Such a reagent can be synthesized e.g. by adding 1 equiv. of methanol or benzyl alcohol to $\mathrm{PCl}_{3}$ and was readily purified by distillation. Subsequent additions of different alcohols yielded products that were further reacted by the same synthetic route mentioned before. ${ }^{[24]}$ We finally moved away from using methanol as a protecting group and switched to benzyl alcohol, as this reagent was readily available in a dry state whereas drying methanol was very time consuming. Furthermore, the deprotection of the benzyl group was possible under mild conditions such as using $\mathrm{CF}_{3} \mathrm{COOH}$ at $25{ }^{\circ} \mathrm{C}$ or by hydrogenation at 1 atm. Our reagent of choice thus became benzyloxydichlorophosphine (BODP, 27). [10]

BODP (27) was first used with motifs such as palmitic alcohol (23), Pet-OH-Pet (24), and Pes-OH-Pes (26) to yield the respective phospholipids $(\mathbf{2 9}, \mathbf{3 0}$, and $\mathbf{3 2}$, Scheme 8).[10] Our main interest, however, was heading towards the synthesis of polymerizable phospholipids and we were able to isolate PanAc-PC-PanAC, the first amine-bearing phospholipid which was accessed via the protected amines $\mathbf{2 5}$ and 31. ${ }^{[25]}$

\section{Vesicle Purification by Ultrafiltration Using a Hollow Fiber Cartridge}

One of the most attractive applications for phospholipids is their formulation into vesicles (liposomes) for drug delivery applications. ${ }^{[26]}$ Such large unilamellar vesicles of $100 \mathrm{~nm}$ diameter (LUVET ${ }_{100}$ ) for release studies are commonly prepared by the thin film method and loaded with a drug followed by an extrusion step through a $100 \mathrm{~nm}$ track-etch polycarbonate membrane. ${ }^{[27]}$ After preparation of the liposomes the internal and external buffers contain equal amounts of the drug and it is necessary to exchange the external buffer. We were using the commonly reported purification by size exclusion chromatography, in our case using a column loaded with Sephadex G50. This works well but does have some limitations. Column conditioning takes around $25 \mathrm{~min}$ for a $40 \mathrm{~mL}$ column and cleaning afterwards takes around the same amount of time. The Sephadex media requires frequent cleaning with $\mathrm{NaOH}$ between uses to maintain a high flow rate. Furthermore, in the case of colorless liposomes, it can be hard to see where the product is eluted from the column, and fraction identification must be done by dynamic light scattering (DLS).

Purification by ultrafiltration using a hollow fiber cartridge is an attractive alternative method and has been used before in, e.g. the preparation of liposome encapsulated cisplatin. ${ }^{[28]}$ It should allow a move towards less labor-intensive purification. We endeavored to purify a LUVET ${ }_{100}$ formulation from the phospholipid Egg-PC. The system we used for the purification of LUVET $_{100}$ comprised a GE Healthcare Hollow Fiber Cartridge (100,000 MW cutoff) and MPLC pump.

All vesicles in this study were prepared by the thin film method. ${ }^{[27]}$ Briefly, a thin film of $30 \mu \mathrm{mol}$ lipid was hydrated with a 5(6)-carboxyfluorescein buffer $(50 \mathrm{mM}$ 5(6)-carboxyfluroescein, $10 \mathrm{mM} \mathrm{NaCl}$, $10 \mathrm{mM}$ HEPES, $\mathrm{pH}$ 7.4), exposed to five freeze thaw cycles, then extruded eleven times through a $100 \mathrm{~nm}$ polycarbonate filter (Whatman, GE Healthcare, USA) above the $\mathrm{T}_{\mathrm{m}}$ of the lipid constituents.

Before the exchange of the external buffer and the removal of debris from the liposome suspension, the hollow fiber cartridge was conditioned with $300 \mathrm{~mL}$ MilliQ water at an inlet flow of $6 \mathrm{~mL} /$ min followed by $300 \mathrm{~mL}$ rinsing buffer (107 mM NaCl, 10 mM HEPES, pH 7.4) using the same conditions.

The liposomes were then injected into the column and the retentate return valve was closed, allowing the liposomes to accumulate in the inner wall side of the hollow fibers. The column was washed with rinsing buffer until there were no further 


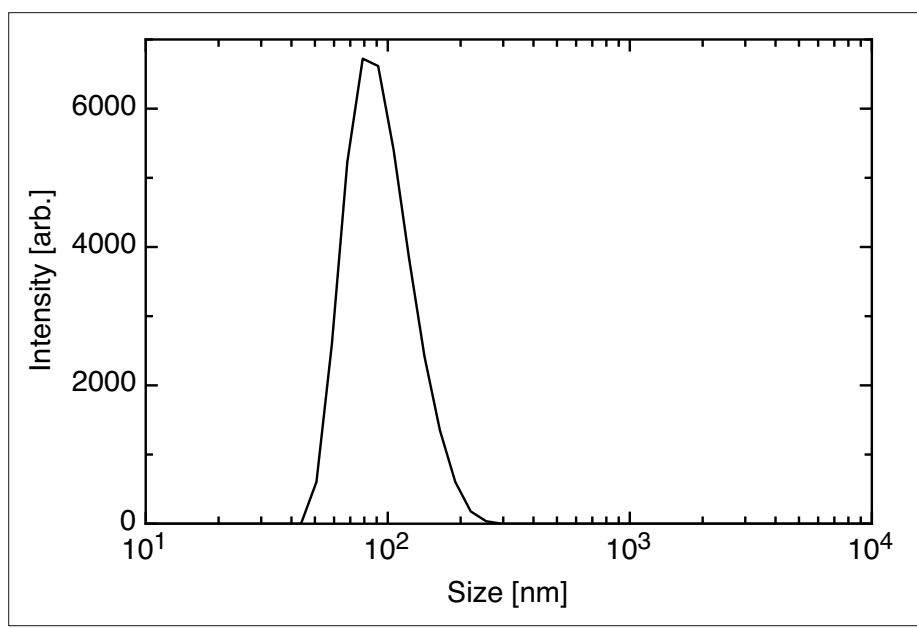

Fig. 1. A uniform population of LUVET 100 was obtained after extrusion and vesicle purification on a hollow fiber cartridge.

traces of carboxyfluorescein in the eluent collected from the outer side of the hollow fibers. The retentate valve was then opened and the liposomes were collected from this outlet by further eluting with rinsing buffer. About $20 \mathrm{~mL}$ of liposome suspension was collected this way and the liposome quality was verified using dynamic light scattering analysis (Fig. 1).

The results from the Egg-PC vesicles showed that $100 \mathrm{~nm}$ particles had indeed been isolated and fluorescence measurements confirmed that external buffer exchange had been effective.

Additionally, concentration of a dilute liposome suspension was achieved by loading it into the hollow fiber cartridge with the retentate return valve closed. In brief, the dilute liposome suspension was loaded into the column and rinsed with rinsing buffer, before the retentate return valve was opened to allow the concentrated liposomes to be collected. A purification using a hollow fiber cartridge provides liposomes of equal quality to results obtained with Sephadex size exclusion chromatog- raphy. Furthermore, with the hollow fiber cartridge ultrafiltration setup it is possible to quickly and easily concentrate vesicle suspensions to a desired concentration.

In conclusion, we gave a brief overview of past and current protocols in phospholipid synthesis and presented the modularity of the phospholipid platform technology. We are convinced that organic synthesis will provide interesting new molecules for studying membranes in the NCCR Chemical Biology. We are looking forward to interesting convergence between the fields of Biology and Chemistry.

\section{Acknowledgement}

We thank the NCCR Chemical Biology for the opportunity to publish in this issue of Chimia.

Received: September 2, 2011

[1] A. Zumbuehl, Chimia 2009, 63, 63.

[2] H. Hirschmann, J. Biol. Chem. 1960, 235, 2762.

[3] J. Chevallier, N. Sakai, F. Robert, T. Kobayashi, J. Gruenberg, S. Matile, Org. Lett. 2000, 2, 1859 .
[4] T. L. Andresen, J. Davidsen, M. Begtrup, O. G. Mouritsen, K. Jørgensen, J. Med. Chem. 2004 47, 1694

[5] L. Clary, C. Santaella, P. Vierling, Tetrahedron 1995, 51, 13073

[6] H. Liu, Z. Zhu, H. Kang, Y. Wu, K. Sefan, W. Tan, Chem. Eur. J. 2010, 16, 3791.

[7] I. A. Fedotenko, P.-L. Zaffalon, F. Favarger, A Zumbuehl, Tetrahedron Lett. 2010, 51, 5382.

[8] Z. Huang, M. R. Jaafari, F. C. Szoka, Angew. Chem. Int. Ed. 2009, 48, 4146.

[9] T. Heimburg, 'Thermal Biophysics of Membranes', Wiley-VCH, 2007.

[10] P.-L. Zaffalon, A. Zumbuehl, Synthesis 2011 , 778.

[11] A. Grün, R. Limpächer, Ber. 1926, 59, 1345.

[12] W. G. Rose, J. Am. Chem. Soc. 1947, 69, 1384.

[13] R. Hirt, R. Berchtold, Pharm. Acta Helv. 1958, $33,349$.

[14] H. Eibl, A. Nicksch, Chem. Phys. Lipids 1978 22,1 .

[15] N. T. Thuong, P. Chabrier, Bull. Soc. Chim. Fr. 1973, 3-4, 667.

[16] O.-Y. Jeon, E. M. Carreira, Org. Lett. 2010, 12, 1772 .

[17] E. E. Nifanteev, D. A. Predvoditelev, V. A. Shin, Mendeleev Chem. J. 1978, 23, 27.

[18] P. Chabrier, N. T. Thuong, M. Chassignol, O. Foussard-Blanpin, J. Bretaudeau, C. Warolin, Ann. Pharm. Fr. 1980, 38, 65

[19] H.-S. Byun, R. K. Erukulla, R. Bittman, J. Org. Chem. 1994, 59, 6495.

[20] S. L. Beaucage, M. H. Caruthers, Tetrahedron Lett. 1981, 22, 1859.

[21] K. S. Bruzik, G. Salamonczyk, W. J. Stec, J. Org. Chem. 1986, 51, 2368.

[22] Y. Xu, S. A. Lee, T. G. Kutateladze, D. Sbrissa, A. Shisheva, G. D. Prestwich, J. Am. Chem. Soc. 2006, 128, 885 .

[23] R. S. Lankalapalli, A. Ouro, L. Arana, A. Gómez-Muñoz, R. Bittman, J. Org. Chem. 2009, $74,8844$.

[24] S. Martin, Tetrahedron Lett. 1988, 29, 3631.

[25] P.-L. Zaffalon, E. Stalder, I. A. Fedotenko, F. Favarger, A. Zumbuehl, Tetrahedron Lett. 2011, $52,4215$.

[26] V. P. Torchilin, Nat. Rev. Drug Discov. 2005, 4, 145.

[27] F. Olson, C. A. Hunt, F. C. Szoka, W. J. Vail, D. Papahadjopoulos, Biochim. Biophys. Acta 1979, 557, 9.

[28] I. Aron, A. Kodre, R. M. Abra, A. Huang, J. J. Vallner, D. D. Lasi, Colloids Surf. B 2004, 33 , 199. 